\title{
Hemangioma hepático gigante asociado a síndrome de Kasabach-Merrit
}

\author{
M. Valdés Mas, M. L. Ortiz Sánchez, J. L. Rodrigo Agudo, M. Miras López, J. A. Pons Miñano \\ y F. Carballo Álvarez
}

Servicio de Aparato Digestivo. Hospital Universitario Virgen de la Arrixaca. El Palmar, Murcia

\section{RESUMEN}

El hemangioma hepático es el tumor benigno más frecuente del hígado. La gran mayoría de ellos son de pequeño tamaño, asintomáticos y con un pronóstico excelente. En raras ocasiones son de mayor tamaño y pueden estar asociados a una coagulopatía de consumo denominada síndrome de Kasabach-Merrit. Presentamos el caso de un paciente varón joven con un hemangioma hepático gigante y hemangiomatosis múltiple asociado al síndrome de Kasabach-Merrit.

Palabras clave: Hemangioma cavernoso. Hemangioma hepático gigante. Síndrome de Kasabach-Merrit.

\begin{abstract}
Hepatic hemangioma is the most frequent liver's tumor. The majority are small, asymptomatic and have an excellent prognosis. Those larger than $5 \mathrm{~cm}$ can be associated to a consumptive coagulopathy called Kasabach-Merrit syndrome. We present a patient with a giant hepatic hemangioma with multiple hemangimatosis associated to Kasabach-Merrit syndrome.
\end{abstract}

Key words: Cavernous hemangioma. Giant hepatic hemangioma. Kasabach-Merrit syndrome.

Valdés Mas M, Ortiz Sánchez ML, Rodrigo Agudo JL, Miras López, Pons Miñano JA, Carballo Álvarez F. Hemangioma hepático gigante asociado a sindrome de Kasabach-Merrit. Rev Esp Enferm Dig 2008; 100: 511-513.

\section{INTRODUCCIÓN}

El hemangioma cavernoso es el tumor mesenquimal benigno más frecuente del hígado (1). Puede presentarse como múltiples lesiones, aunque lo más frecuente es que se trate de una lesión única. Son poco frecuentes, llegándose a presentar entre un 1,2 y un $5 \%$ de las autopsias $(1,2)$.

La mayoría son menores de $5 \mathrm{~cm}$. Cuando miden más de $5 \mathrm{~cm}$ se consideran gigantes y pueden estar asociados a coagulación intravascular diseminada. Generalmente son asintomáticos y tienen un pronóstico excelente $(1,3)$.

Aquí describimos el caso de un hemangioma gigante hepático con hemangiomatosis múltiple asociado a síndrome de Kasabach-Merrit en un paciente inmigrante joven.

Recibido: 22-04-08

Aceptado: 06-05-08.

Correspondencia: Mariano Valdés Mas. Unidad de Hepatología. Hospital Universitario Virgen de la Arrixaca. Ctra. Madrid-Cartagena, s/n. 30120 El Palmar, Murcia.e-mail: marianovaldesmas@yahoo.es

\section{CASO CLÍNICO}

Paciente varón de 32 años que acude al servicio de urgencias por hepatomegalia gigante dolorosa tras traumatismo lumbar, acompañado de distensión abdominal. Entre sus antecedentes destaca una intervención quirúrgica en Marruecos de un hemangioma hepático hace año y medio, del cual no tenemos más información (Fig. 1). Sin otros antecedentes médicos ni familiares de interés. El paciente tiene una situación basal normal para su edad. A la exploración física presenta una tensión arterial de 140/70 con una frecuencia cardiaca de $72 \mathrm{lpm}$ y una temperatura de $36,5^{\circ} \mathrm{C}$. Presenta un soplo sistólico en foco mitral, auscultación pulmonar con murmullo vesicular normal y un abdomen distendido con gran hepatomegalia dolorosa que llega hasta ambas fosas iliacas sin esplenomegalia palpable y con peristaltismo normal.

En los datos analíticos destaca una hemoglobina de $10,4 \mathrm{~g} / \mathrm{dl}$, hematocrito 32,5\%; VCM $82 \mathrm{fl} ; \mathrm{HCM} 26,3 \mathrm{pg}$; plaquetas $144 \times 10^{3} / \mu \mathrm{l}$; leucocitos $3.700 / \mathrm{ul}$ (N: 83,6\%); haptoglobina sérica 77 mg/dl. Bioquímica: GOT 33 U/l; 


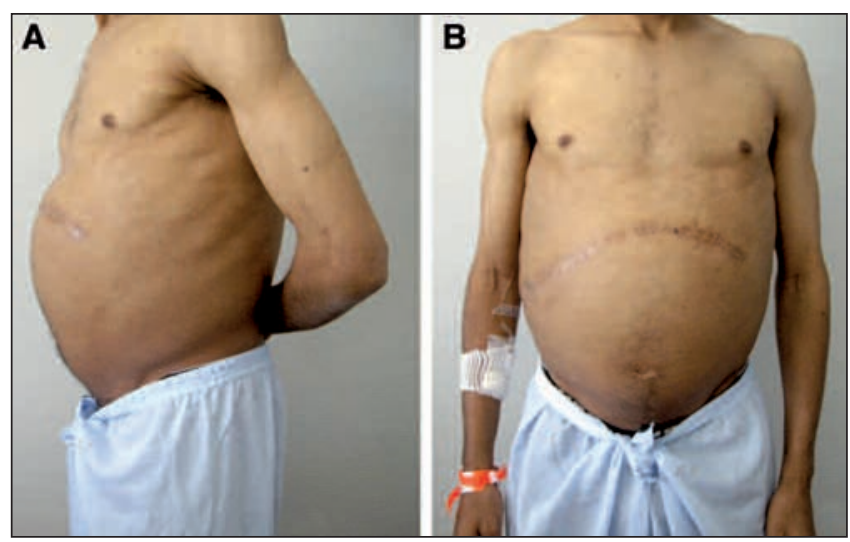

Fig. 1. Fotografía del paciente. Gran distensión abdominal y cicatriz de cirugía previa.

GPT 21 U/l; FA 143 U/1; GGT 217 U/l. Bilirrubina total $1,5 \mathrm{mg} / \mathrm{dl}$; bilirrubina directa: $1 \mathrm{mg} / \mathrm{dl}$. Creatinina 0,93 $\mathrm{mg} / \mathrm{dl}$. Iones y amilasa normales. Serología de virus de la hepatitis B, C, VIH y LUES negativos.

En el estudio de coagulación presentaba una actividad de protrombina del $52 \%$, un dímero D que excede los $2.500 \mathrm{ng} / \mathrm{ml}$, TTPA de 38 segundos, fibrinógeno de 53 $\mathrm{mg} / \mathrm{dl}$, plasminógeno funcional del $48 \%$ e inhibidor de la plasmina del $57 \%$, todo ello siendo compatible con síndrome de Kasabach-Merrit.

La radiografía de tórax mostró una elevación de ambos hemidiafragmas secundaria a la hepatomegalia, sin cardiomegalia ni infiltrados pulmonares (Fig. 2). En la simple de abdomen se aprecia gran hepatomegalia que desplaza asas intestinales a fosa iliaca izquierda (Fig. 2). Ecocardiografía con un corazón estructural y funcionalmente normal, salvo por compresión extrínseca de cavidades derechas por hepatomegalia que no origina compromiso hemodinámico.

Tras su llegada a urgencias, teniendo en cuenta sus antecedentes, se decide realizar TC abdominal para valorar sangrado secundario a rotura traumática del hemangioma, apreciándose: hígado muy aumentado de tamaño con densidad heterogénea, con múltiples imágenes nodulares hipodensas con captación periférica de contraste. Presen-
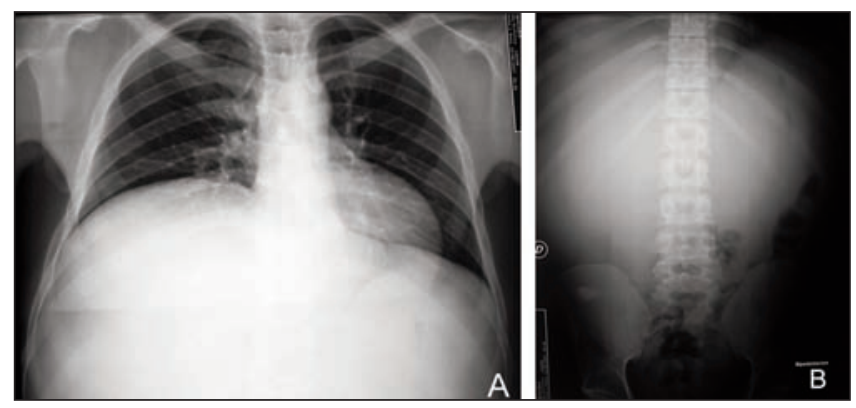

Fig. 2. A: Radiografía de tórax en la que se aprecia elevación de ambos hemidiafragmas; B: Radiografía simple de abdomen con gran hepatomegalia y desplazamiento de asas intestinales. taba además gran masa hipodensa dependiente del caudado, de aspecto lobulado, de un tamaño aproximado de 17 x $7 \mathrm{~cm}$ (T x AP), que presenta patrón de captación de contraste similar a las anteriores y que desplaza en sentido caudal asas intestinales, páncreas, bazo y riñones, sin observar alteraciones significativas en estas estructuras. Pequeña cantidad de líquido libre en Douglas. Todo ello es sugestivo de hemangioma cavernoso hepático. El parénquima hepático parece respetado a nivel del segmento VII (Fig. 3B).

Estos hallazgos se correlacionaron con los ecográficos, confirmando la sospecha diagnóstica de hemangiomatosis múltiple hepática con hemangioma gigante de 17 × 7 $\mathrm{cm}$ y ascitis (Fig. 3C).

Ante la sospecha de rotura traumática del mismo y debido a la estabilidad hemodinámica y analítica del enfermo, se decide realizar angio-TC para valorar la presencia de sangrado activo que obligó a una arteriografía con embolización selectiva, que descarta sangrado activo. Se evidencia compresión de la vena renal izquierda, con formación de colaterales a retroperitoneo (Fig. 3A).

Por todo ello, confirmamos el diagnóstico de hemangioma hepático gigante con hemangiomatosis y síndrome de Kasabach-Merritt asociado.

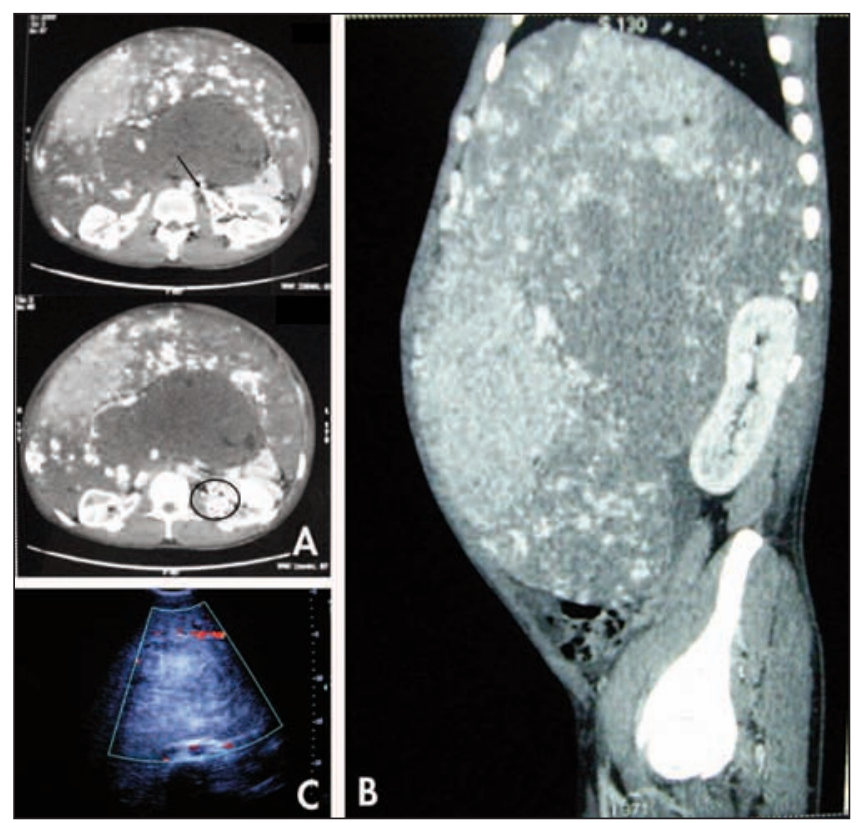

Fig. 3. A: Angio-TC abdominal en la que se aprecia estenosis de la vena renal izquierda (flecha) con aparición de colaterales (círculo); B: Reconstrucción de TC abdominal; y C: Ecografía abdominal con gran vascularización periférica.

\section{DISCUSIÓN}

El hemangioma hepático consiste en una malformación vascular hepática de etiología no bien definida con una prevalencia que va desde un 0,5 hasta un $20 \%$ (4). 
Generalmente suelen ser solitarios, pero pueden presentar múltiples lesiones en ambos lóbulos hepáticos en más del $40 \%$ de los pacientes (5). La mayoría de los hemangiomas tiene un tamaño menor de $5 \mathrm{~cm}$; aquellos cuyo tamaño es superior a $5 \mathrm{~cm}$ se denominan gigantes (6).

Generalmente son asintomáticos y tienen un pronóstico excelente. Los síntomas son más frecuentes en lesiones superiores a $4 \mathrm{~cm}$. El síntoma que más destaca es el dolor abdominal en el $54 \%$ de estos pacientes (7). Se trata de un dolor localizado en hipocondrio derecho y que probablemente es secundario a trombosis o sangrado del tumor, así como de la posible inflamación de la cápsula de Glisson. Otros síntomas menos comunes son las náuseas, anorexia y saciedad precoz; todos ellos secundarios a la compresión de los órganos circundantes $(4,6)$.

En el caso que presentamos nos encontramos que, a pesar del tamaño del hígado, el paciente se encuentra asintomático sin alteraciones del hábito intestinal. Sí encontramos, en cambio, signos de probable compresión de la vena renal izquierda con colaterales que drenan a retroperitoneo.

En síndrome de Kasabach-Merritt es una coagulopatía de consumo en niños que ha sido descrita asociada a hemangiomas gigantes. Cursa con severa trombocitopenia, hipofibrinogenemia, elevación de los productos de degradación del fibrinógeno y fragmentación de los glóbulos rojos. El caso presenta a un paciente que ha permanecido asintomático hasta la edad adulta y que presenta una coagulopatía de consumo asociada.

La historia natural del hemangioma hepático no se conoce completamente. La mayoría de los pacientes permanece estable a lo largo del tiempo $(4,8,9)$. Únicamente se aprecia un aumento de tamaño de forma ocasional, llegando a requerir resección quirúrgica (10-12). La ruptura espontánea es rara incluso en hemangiomas gigantes.

El manejo de estos pacientes va a depender del tamaño del hemangioma y de la sintomatología. En pacientes asintomáticos, la actitud es expectante más incluso en hemangiomas menores de 1,5 cm (7). En pacientes con síntomas sugestivos de compresión extrínseca se debe considerar la intervención quirúrgica, siendo más indicada la enucleación frente a la resección, debido a que presenta menor sangrado en el postoperatorio $(13,14)$. El trasplante hepático se llevará a cabo en pacientes sintomáticos con lesiones irresecables o en caso de un hemangioma gigante con hemangiomatosis y asociados al síndrome de Kasabach-Merrit (15-17). En determinados casos se ha probado la eficacia de la embolización transarterial de los hemangiomas con mejoría rápida de la coagulopatía de consumo (18).

En nuestro caso, debido al importante tamaño que presentaba el hígado asociado al síndrome de Kasabach-Merrit, hemos planteado al paciente la posibilidad del trasplante hepático.

\section{BIBLIOGRAFÍA}

1. Díez Redondo P, Velicia Llames R, Caro-Patón A. Giant cavernous hemangiomas of the liver. Rev Esp Enferm Dig 2004; 96 (9): 665-6.

2. Ruiz Guinaldo A, Martín Herrera L, Roldán Cuadra R. Hepatic tumors in patients with cirrhosis: an autopsy study. Rev Esp Enferm Dig 1997; 89 (10): 771-80.

3. Alvarez Pérez JA, Baldonedo Cernuda RF, Palacios Fernández E. Giant hepatic cavernous hemangioma. Rev Esp Enferm Dig 1994; 85 (5): 403-4.

4. Gandolfi L, Leo P, Solmi L, et al. Natural history of hepatic haemangiomas: clinical and ultrasound study. Gut 1991;32: 677.

5. Tait N, Richardson AJ, Muguti G, Little JM. Hepatic cavernous haemangioma: A 10 year review. Aust N Z J Surg 1992; 62: 521.

6. Adam YG, Huvos AG, Fortner JG. Giant hemangiomas of the liver. Ann Surg 1970; 172: 239

7. Farges O, Daradkeh S, Bismuth H. Cavernous hemangiomas of the liver: are there any indications for resection? World J Surg 1995; 19 : 19.

8. Yamagata M, Kanematsu T, Matsumata T, et al. Management of haemangioma of the liver: comparison of results between surgery and observation. Br J Surg 1991; 78: 1223.

9. Mungovan JA, Cronan JJ, Vacarro J. Hepatic cavernous hemangiomas: lack of enlargement over time. Radiology 1994; 191: 111.

10. DuPre CT, Fincher RM. Case report: cavernous hemangioma of the liver. Am J Med Sci 1992; 303: 241.

11. Yoshida J, Yamasaki S, Yamamoto J, et al. Growing cavernous haemangioma of the liver: 11-fold increase in volume in a decade. J Gastroenterol Hepatol 1991; 6: 414.

12. Pietrabissa A, Giulianotti P, Campatelli A, et al. Management and follow-up of 78 giant haemangiomas of the liver. Br J Surg 1996; 83: 915.

13. Bornman PC, Terblanche J, Blumgart RL, et al. Giant hepatic hemangiomas: diagnostic and therapeutic dilemmas. Surgery 1987; 101: 445 .

14. Popescu I, Ciurea S, Brasoveanu V, et al. Liver hemangioma revisited: current surgical indications, technical aspects, results. Hepatogastroenterology 2001; 48: 770 .

15. Longeville JH, de la Hall P, Dolan P, et al. Treatment of a giant haemangioma of the liver with Kasabach-Merritt syndrome by orthotopic liver transplant a case report. HPB Surg 1997; 10: 159.

16. Tepetes K, Selby R, Webb M, et al. Orthotopic liver transplantation for benign hepatic neoplasms. Arch Surg 1995; 130: 153.

17. Russo MW, Johnson MW, Fair JH, Brown RS Jr. Orthotopic liver transplantation for giant hepatic hemangioma. Am J Gastroenterol 1997; 92: 1940 .

18. Malagari K. Transarterial embolization of giant liver hemangiomas associated with Kasabach-Merrit syndrome: a case report. Acta Radiol 2007; 48 (6): 608-12. 